\title{
Novel Negative Pressure Helmet Reduces Aerosolized Particles in a Simulated Prehospital Setting
}

\author{
Nathaniel Hunt, MD; ${ }^{1,2}$ (1) Spencer Masiewicz, DO; ${ }^{3}$ Logan Herbert, MD; ${ }^{1}$ Benjamin Bassin, MD; ${ }^{1,2,4}$ \\ Christine Brent, MD; $;^{1,2,4}$ Nathan L. Haas, MD; ${ }^{1,2,4}$ Mohamad Hakam Tiba, MD; ${ }^{1,2}$ \\ Jon Lillemoen, CIH; ${ }^{5}$ Mark J. Lowell, MD; ${ }^{1,2}$ Isabel Lott, BS; ${ }^{6}$ Matthew Basinger, MD; ${ }^{1}$ \\ Graham Smith, MD; ${ }^{1,2}$ Kevin R. Ward, MD ${ }^{1,2}$
}

1. Department of Emergency Medicine, University of Michigan, Ann Arbor, Michigan USA

2. Michigan Center for Integrative Research in Critical Care, Ann Arbor, Michigan USA

3. Department of Emergency Medicine, Northeast Georgia Medical Center, Gainesville, Georgia USA

4. Division of Critical Care, Department of Emergency Medicine, University of Michigan, Ann Arbor, Michigan USA

5. University of Michigan Environment, Health and Safety, Ann Arbor, Michigan USA

6. University of Michigan Medical School, Ann Arbor, Michigan USA

Correspondence:

Nathaniel Hunt, MD Clinical Instructor Department of Emergency Medicine University of Michigan Taubman Center, B13541500 E. Medical Center Dr, SPC 5303 Ann Arbor, Michigan 48109-5303 USA

E-mail: nateryan@med.umich.edu

Conflicts of interest/funding: Kevin Ward has intellectual property regarding the AerosolVE Helmet through the University of Michigan which has been licensed to Inspire Rx LLC. Kevin Ward, Ben Bassin, and Nathan Haas have equity in Inspire Rx LLC. Ben Bassin is the Chief Medical Officer for Inspire Rx LLC. The remaining authors have no competing interests.

Keywords: aerosol; COVID-19; helmet; mitigation; prehospital

\section{Abbreviations:}

AGP: aerosol-generating procedure COVID-19: coronavirus disease 2019

CPAP: continuous positive airway pressure EMS: Emergency Medical Services HEPA: high efficiency particulate air filter

\begin{abstract}
Background/Objective: The coronavirus disease 2019 (COVID-19) pandemic has created challenges in maintaining the safety of prehospital providers caring for patients. Reports have shown increased rates of Emergency Medical Services (EMS) provider infection with COVID-19 after patient care exposure, especially while utilizing aerosol-generating procedures (AGPs). Given the increased risk and rising call volumes for AGP-necessitating complaints, development of novel devices for the protection of EMS clinicians is of great importance.

Drawn from the concept of the powered air purifying respirator (PAPR), the AerosolVE helmet creates a personal negative pressure space to contain aerosolized infectious particles produced by patients, making the cabin of an EMS vehicle safer for providers. The helmet was developed initially for use in hospitals and could be of significant use in the prehospital setting. The objective of this study was to determine the efficacy and safety of the helmet in mitigating simulated infectious particle spread in varied EMS transport platforms during AGP utilization.
\end{abstract}

Methods: Fifteen healthy volunteers were enrolled and distributed amongst three EMS vehicles: a ground ambulance, a medical helicopter, and a medical jet. Sodium chloride particles were used to simulate infectious particles, and particle counts were obtained in numerous locations close to the helmet and around the patient compartment. Counts near the helmet were compared to ambient air with and without use of AGPs (non-rebreather mask $[\mathrm{NRB}]$, continuous positive airway pressure mask [CPAP], and high-flow nasal cannula [HFNC]).

Results: Without the helmet fan on, the particle generator alone and with all AGPs produced particle counts inside the helmet significantly higher than ambient particle counts. With the fan on, there was no significant difference in particle counts around the helmet compared to baseline ambient particle counts. Particle counts at the filter exit averaged less than one despite markedly higher particle counts inside the helmet.

HFNC: high-flow nasal cannula

HHFNC: heated high-flow nasal cannula

HVAC: heat, ventilation, air conditioning

NRB: non-rebreather mask

O2: oxygen

PAPR: powered air purifying respirator

PPE: personal protective equipment

Received: September 20, 2021

Revised: October 23, 2021

Accepted: October 31, 2021

doi:10.1017/S1049023X22000103

(C) The Author(s), 2022. Published by Cambridge University Press on behalf of the World Association for Disaster and Emergency Medicine. This is an Open Access article, distributed under the terms of the Creative Commons Attribution licence (https://creativecommons.org/licenses/by/4.0/), which permits unrestricted re-use, distribution, and reproduction in any medium, provided the original work is properly cited. 
Conclusion: Given the risk to EMS providers by communicable respiratory diseases, development of devices to improve safety while still enabling use of respiratory therapies is of paramount importance. The AerosolVE helmet demonstrated efficacy in creating a negative pressure environment and provided significant filtration of simulated respiratory droplets, thus making the confined space of transport vehicles potentially safer for EMS personnel.

Hunt N, Masiewicz S, Herbert L, Bassin B, Brent C, Haas NL, Tiba MH, Lillemoen J, Lowell MJ, Lott I, Basinger M, Smith G, Ward KR. Novel negative pressure helmet reduces aerosolized particles in a simulated prehospital setting. Prehosp Disaster Med. 2022;37(1):33-38.

\section{Introduction}

The coronavirus disease 2019 (COVID-19) pandemic has created challenges in maintaining the safety of Emergency Medical Services (EMS) providers while also allowing the provision of maximally aggressive respiratory therapies for patient care. Many patients transported by EMS require respiratory therapies, including nebulized medications, continuous positive airway pressure (CPAP) non-invasive ventilation, or heated high-flow nasal cannula oxygen therapy (HHFNC). These treatments are considered aerosol-generating procedures (AGPs) and thus have the potential to increase spread of infectious viral agents, such as COVID-19.,2 Use of such therapies places EMS providers at particular risk given the small physical confines of an ambulance or helicopter and lack of ventilation systems designed to mitigate aerosolization of infectious agents. Consequently, many EMS agencies have limited the use of these AGPs despite the potential patient benefit.

Prior data indicate EMS providers can be actively infected during transport of COVID-19 patients. At the start of the pandemic in New York City (New York USA), there was a significant increase in 9-1-1 calls for respiratory complaints, cardiovascular complaints, and cardiopulmonary arrest. There was also an increase in high-acuity life-threatening calls resulting in increased exposure of EMS staff to aerosolizing procedures and augmented risk of contracting COVID-19. ${ }^{3}$ Data from King County, Washington (USA) demonstrated that $16.3 \%$ (182 of 1,115) of encounters for COVID-19 had one or more aerosolizing procedures performed by EMS. Incidence of COVID-19 in EMS personnel during that period was 0.57 infections $/ 10,000$ person-days. ${ }^{4}$ Out of 274 EMS encounters with COVID-19-confirmed patients, there were 151 person-exposures among 129 EMS providers, resulting in 981 quarantine days with a $0.4 \%$ positive test rate. ${ }^{5}$ Additionally, early in the pandemic testing of 3,326 first responders in Arizona (USA), $1.5 \%$ tested positive for COVID-19. ${ }^{6}$ Heinzerling, et al found that $67 \%$ of medical personnel who assisted COVID-19infected patients undergoing nebulization developed infection themselves. The authors noted that since the virus has spread within the hospital environment, it should be assumed that chances of getting infected are significantly higher in the limited space of an ambulance. ${ }^{7}$ The increasing exposure to respiratory complaints in the EMS field necessitates updated personal protective equipment (PPE) to minimize prehospital exposures, especially during AGPs. The risk of COVID-19 infection and/or mandatory quarantine following a significant exposure can be mitigated by improving prehospital PPE.
During the COVID-19 pandemic, the University of Michigan Department of Emergency Medicine (Ann Arbor, Michigan USA), Michigan Center for Integrative Research in Critical Care (M-CIRCC; Ann Arbor, Michigan USA), and the College of Engineering (Ann Arbor, Michigan USA) collaborated with a local manufacturing company to develop a device capable of mitigating these risks. The device (AerosolVE Helmet; Inspire Rx LLC; Ann Arbor, Michigan USA) consists of a helmet equipped with an air purifying filter, based on the common powered air purifying respirator (PAPR), but is uniquely re-engineered to reverse air flow. This design allows room air to be pulled into the helmet which is then passed through a high efficiency particulate air (HEPA) filter before being released back into the ambient environment (Figure 1). This creates a negative pressure environment within the helmet, thus infectious particles produced and aerosolized by the patient and/or any AGP are contained within the helmet and filtered before release into ambient air. The device is designed to be able to accommodate use of HHFNC, CPAP/ BiPAP, and nebulized aerosol therapies. The secured face shield can be opened for emergency access to the patient's face. The lower portion (bib) of the helmet is loosely fitted to allow large volumes of room air to be pulled through the helmet and to allow provider and patient access to the patient's face by the patient or provider without having to open the face shield. The HEPA filter is integral to the motor (fan) and is designed to pull air through the helmet at 220 liters/minute. When compared to current recommendations for hospital negative pressure rooms of at least 12 air exchanges per hour, ${ }^{8}$ the helmet produces 840 air exchanges per hour, or 70 -times more air exchanges than current hospital room recommendations. The helmet, shroud, and hose are designed to be disposable. The motor and filter are designed to be reusable with filter life of 12 months or until the filter alarm activates. An alarm exists to detect when flow decreases to 170 liters/minute.

The helmet and a similar negative pressure tent were initially developed for use in hospitals. Previous publications have demonstrated their efficacy in reducing air particle counts, likely improving the safety of providers caring for patients with communicable respiratory illnesses. ${ }^{9-12}$

The prehospital transport environment varies significantly from a hospital room, and environments between transport vehicle platforms (ie, jet versus helicopter versus ground ambulance) are also not equivocal. Thus, testing the device in each different transport platform is integral to ensure efficacy and safety.

The objective of this study was to test the effects of the negative pressure helmet device on air particle counts in healthy volunteers undergoing a variety of AGPs in simulated prehospital settings. It is hypothesized the AerosolVE helmet would prevent increases in air particle counts in the ambient cabin air.

\section{Methods}

This was an open-label study of the efficacy of the AerosolVE helmet and filtration system. Fifteen healthy volunteers were enrolled, twelve men and three women, and were distributed amongst three transport platforms: a LearJet 75 configured for medical transport, a Eurocopter EC155 medical helicopter, and a Ford E450 modular ambulance. While not a requirement, all volunteers had been fully vaccinated for COVID-19 prior to participation. Each participant was screened for signs and symptoms of COVID-19 or other respiratory illness prior to enrollment. Sodium chloride particles, generated by a TSI 8026 particle generator (TSI Inc; Shoreview, Minnesota USA), were emitted near the subjects' mouths to 


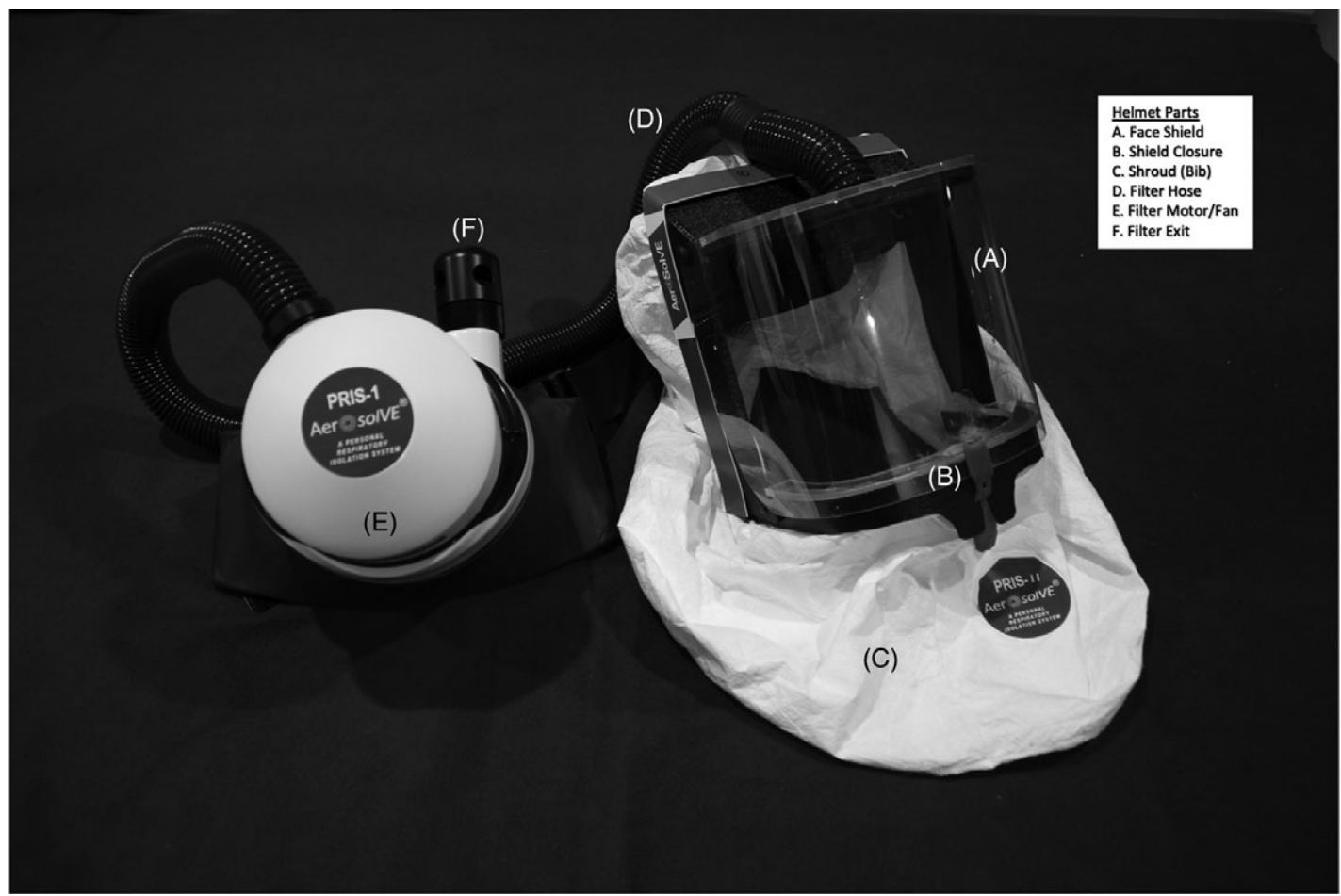

Hunt $\odot 2022$ Prehospital and Disaster Medicine

Figure 1. AerosolVE Helmet.

Note: The AerosolVE helmet is a negative pressure device modeled after a PAPR. The clear face shield (A) allows for good visibility and can be opened by the red tab (B) for immediate access to the patient's face. The filter and motor (E) can be held or worn by a belt. Abbreviation: PAPR, powered air purifying respirator.

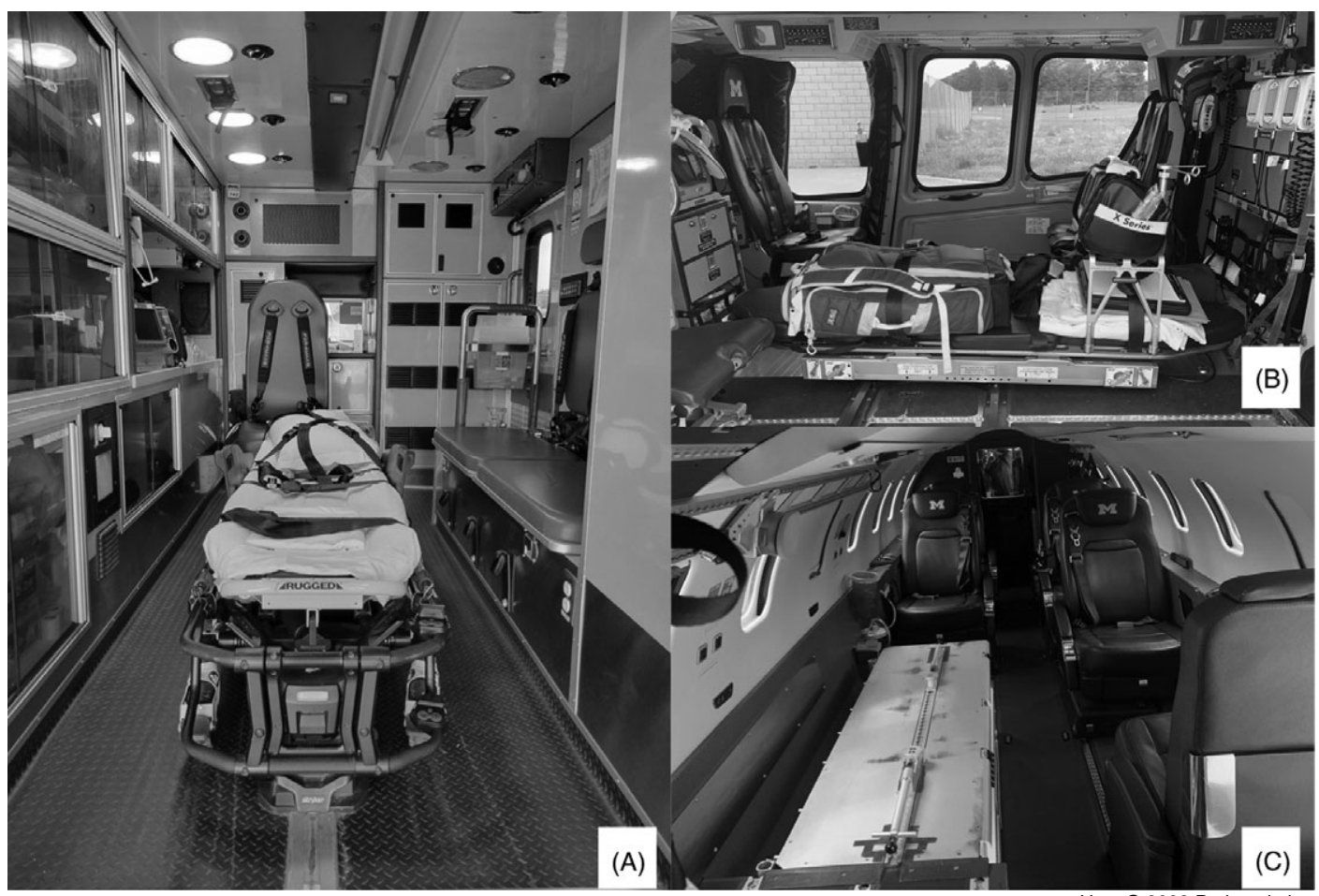

Hunt () 2022 Prehospital and Disaster Medicine

Figure 2. EMS Transport Platforms.

Note: Patient compartment view of each transport platform. Driver/pilot compartment not visible. A) Ground Ambulance. B) Medical Helicopter. C) Medical Jet.

Abbreviation: EMS, Emergency Medical Services. 


\begin{tabular}{|c|c|c|c|c|}
\hline & Ambient & Close to Helmet & Inside Helmet Motor On & Inside Helmet Motor Off \\
\hline \multicolumn{5}{|c|}{ Ambulance } \\
\hline Baseline & 4057 (3581) & $4063(2961)$ & $5529(2805)$ & - \\
\hline CPAP & $3567(2846)$ & $3616(3204)$ & $5554(5820)$ & $75862(22350)^{a}, b_{,}, c$ \\
\hline HFNC & 3755 (3057) & 3636 (3387) & $4653(2113)$ & $85270(30332) a, b c$ \\
\hline \multicolumn{5}{|c|}{ Helicopter } \\
\hline NRB & $5419(3101)$ & $4579(2220)$ & $17880(23266)$ & $142670(20558) a, b, c$ \\
\hline CPAP & 5939 (3338) & $6072(2786)$ & $13138(13424)$ & $101466(41614), a, b, c$ \\
\hline HFNC & $5666(3087)$ & $5736(2798)$ & $18247(16798)$ & $164699(16970)^{a}, \mathrm{~b}, \mathrm{c}$ \\
\hline \multicolumn{5}{|l|}{ Jet } \\
\hline Baseline & $1522(1312)$ & $1726(1009)$ & $25118(20242)$ & - \\
\hline
\end{tabular}

Table 1. Mean (SD) Particle Counts for Each Transport Platform and AGP

Hunt $\odot 2022$ Prehospital and Disaster Medicine

Note: Mean (standard deviation) particle counts for each transport platform and each AGP tested around the cabin, close to the helmet, inside the helmet with the motor on and off.

Abbreviations: AGP, aerosol-generating procedure; NRB, non-rebreather mask; CPAP, continuous positive airway pressure; HFNC, high-flow nasal cannula.

${ }^{a}$ Denotes significant difference between "Inside Helmet Motor off" and "Ambient" $(\mathrm{P}<.0001)$.

b Denotes significant difference between "Inside Helmet Motor off" and "Close to Helmet" $(\mathrm{P}<.0001)$.

${ }^{\mathrm{c}}$ Denotes significant difference between "Inside Helmet Motor off" and "Inside Helmet Motor on" $(\mathrm{P}<.0001)$.

simulate bioaerosol generation associated with viral respiratory infections and to maximally test the system. A TSI 3007 condensation particle counter (TSI Inc; Shoreview, Minnesota USA) was used to detect and quantify air particle counts at different locations, including particle leakage from the helmet and particle concentration inside the helmet. The device is capable of detecting and quantifying particles in the range of 0.01 to $>1.0 \mu \mathrm{m}$ which would include the size of the COVID-19 virus $(0.1 \mu \mathrm{m}){ }^{13}$ This study was approved by the University of Michigan Institutional Review Board and required written consent (protocol \# HUM00192223).

Baseline ambient air particle counts were obtained in the closed cabin of the selected vehicle without oxygen $(\mathrm{O} 2)$ devices on and without active particle generation. This was to determine the ambient particle counts present related to dust and other environmental particles. The participant was then seated in the standard patient transport position and the helmet was placed on the participant. The helmet was in the standard configuration as provided from the manufacturer. The particle generator was inserted into the helmet from underneath the helmet's lower bib and turned on. This was meant to simulate active expiration and aerosolization of infectious particles from a patient. Particle counts were obtained in numerous locations around the helmet and in various locations about the transport platform's cabin (Figure 2A-C). Once counts had been obtained without use of an $\mathrm{O} 2$ device, the procedure was repeated with the participant wearing a non-rebreather mask $(\mathrm{NRB})$ at $15 \mathrm{~L} /$ minute $\mathrm{O} 2$ flow, a CPAP mask with pressure of $5 \mathrm{~cm}$ of water, and a high-flow nasal cannula (HFNC) with $30 \mathrm{~L} /$ minute of flow. These tests were performed in succession without any break in the cabin to ensure no inadvertent disruption of ambient particle levels (eg, dust in the outside air entering the cabin that would affect interpretation of subsequent particle counts). The participant had to briefly remove the helmet for each change of $\mathrm{O} 2$ delivery device. At each testing location, ten particle counts were obtained and the mean recorded. This was to account for respiratory variation and other environmental factors that may cause small shifts in particle counts at that location (eg, participants breathing in particles resulting in a momentary reduction in counts inside the helmet). Primary outcome was the difference in ambient particle counts and counts close to the helmet compared to counts inside the helmet with the filter motor on.

\section{Statistical Analysis}

Data are presented as mean (standard deviation). One way ANOVA was used to compare the primary outcome (particle count inside helmet with filter on) to particle count in the ambient air and the environment close to the helmet. Statistical significance was considered as $\alpha=0.05$. All data were analyzed using PRISM 9 (GraphPad Software; San Diego, California USA).

\section{Results}

Table 1 and Figure 3 present the mean air particle counts with and without the use of each $\mathrm{O} 2$ delivery device. Without the helmet fan on, the particle generator alone and all AGPs produced particle counts inside the helmet significantly higher than ambient particle counts. With the helmet fan on, particle counts near the helmet showed no significant elevation compared to baseline ambient particle counts during the use of the particle generator alone or with use of any of the AGPs. Additionally, particle counts were significantly lower in the helmet while the motor was on compared to when the motor was off for each $\mathrm{O} 2$ delivery device. Table 2 demonstrates that particle counts taken at the fan exit (post-HEPA filter) were reduced to nearly zero compared to counts inside the helmet. Interestingly, with the face shield up (which could be necessary for emergent access), there was no significant difference in 


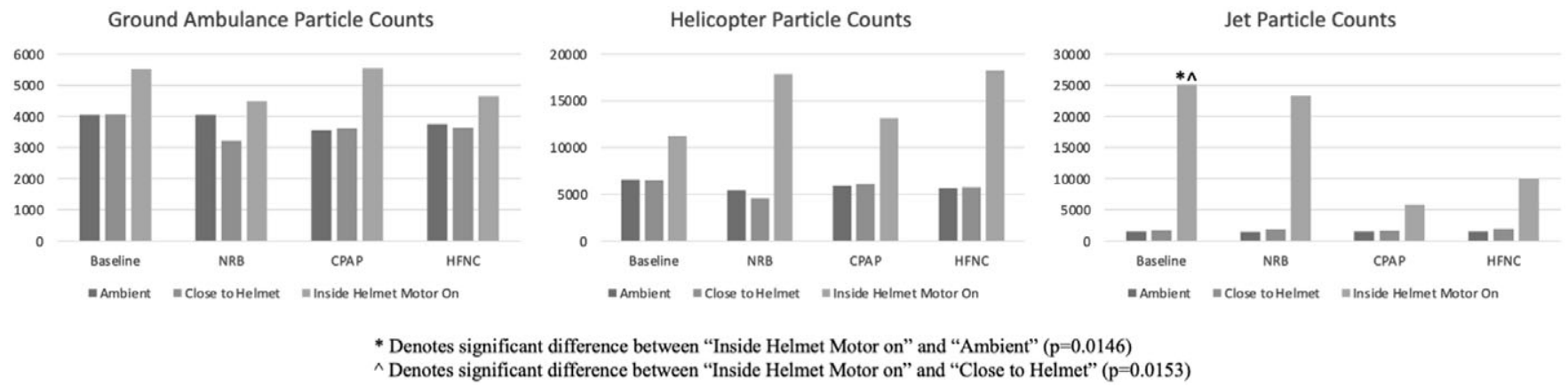

Figure 3. Mean Particle Counts by Platform and AGP.

Note: Mean particle counts by EMS transport platform and by AGP. Scale differs by platform.

Abbreviations: AGP, aerosol-generating procedure; EMS, Emergency Medical Services; NRB, non-rebreather mask; CPAP, continuous positive airway pressure; HFNC, high-flow nasal cannula.

\begin{tabular}{|l|c|c|c|}
\hline $\mathrm{O}_{\mathbf{2}}$ Device & Ambulance & Helicopter & Jet \\
\hline Baseline & $0.6(0.41,0.87)$ & $0.8(0.46,1.1)$ & $0.8(0.42,1.14)$ \\
\hline NRB & $0.6(0.31,0.89)$ & $0.7(0.35,0.77)$ & $1.4(-0.54,3.3)$ \\
\hline CPAP & $0.6(0.28,1)$ & $0.7(0.54,0.9)$ & $0.9(0.47,1.25)$ \\
\hline HFNC & $0.6(0.19,1.05)$ & $0.8(.44,1.16)$ & $0.6(0.48,0.72$ \\
\hline \multicolumn{4}{|c|}{ Hunt $\odot$ 2022 Prehospital and Disaster Medicine }
\end{tabular}

draw of air into the helmet prevents loss of particles into the patient compartment. These results show this device not only filters air but also effectively contains simulated infectious particles, preventing disbursement and potentially reducing risk to providers during AGPs.

The testing protocol specifically excluded the use of a nebulizer, a common respiratory care device used in the treatment of respiratory distress in the prehospital setting. Previous testing had shown similar generation of aerosolized particles from the particle generator and the nebulizer. ${ }^{9}$ The authors chose to use only the generator for simplicity. They also chose not to test participants in various transport positions. Given the dynamics of the AerosolVE device (ie, pulling environmental air into the helmet), any increase in gaps under the shroud would allow more air flow and would likely improve the air exchange; thus, various positions would be unlikely to have a significant negative impact on results. Lastly, the authors elected not to modulate the air flow (ie, heat, ventilation, air conditioning $[\mathrm{HVAC}])$ in the patient compartment of the ambulance as they wanted to specifically test the device in a "worst-case scenario" (no air exchange present). Any HVAC use would only serve to improve the ambient air quality. In addition, for the helicopter and jet, the authors would not have been able to test the device with HVAC running as this requires the aircraft to be running. The baseline ambient particle counts, as a result from dust or exhaust, were too high to measure any difference generated by the particle generator.

Despite the protracted nature of the current COVID-19 pandemic, there are no negative pressure or containment technologies approved for prehospital patient transport use. While a number of negative pressure/isolation "tents" have gained Emergency Use Authorization (EUA) for use within hospitals, none to the authors' knowledge are approved for prehospital use. Furthermore, some of these rely on regular hospital wall suction to produce a negative pressure within the tent. The efficacy of this level of negative pressure and flow to reduce AGP increases in ambient particle counts back to baseline has not been reported.

Due to the risk of contamination, many EMS systems have reduced their transport of patients with known or highly suspected COVID-19 infections or modified protocols to reduce risk to providers (eg, using supraglottic airways rather than oral endotracheal intubation, metered-dose inhalers rather than nebulizer treatment). While the ability was tested of the AerosolVE Helmet to reduce AGP particle counts to baseline, the device can likely 
provide benefit during transport of any patient with unknown COVID-19 status, regardless of symptoms, as a means to reduce EMS provider exposure and allow for maximal patient therapy. It could also allow transport of multiple infected patients without increasing the risks of infection to EMS providers.

Similar challenges exist in the transport of military patients with COVID-19 as have been described in the civilian EMS sector, and the likelihood of a multiple-patient transport environment is higher in the military sector. Negative pressure transport conexes have been designed to transport multiple infected individuals. ${ }^{14}$ Use of devices like the helmet described in this report may offer additional options.

\section{Limitations}

This study was conducted on healthy volunteers who were breathing normally (not coughing) with simulated infectious droplets dispersed within the helmet. As this study was to determine efficacy of the device's ability to prevent increases in cabin particle counts and particle counts near the subject in the EMS transport environment, it utilized a small convenience sample of volunteers rather than a large population. Given the efficiency of the device in its ability to filter particles, it is unlikely that additional test participants would have made a significant difference in the results obtained. Future studies will be required examining the device and particle counts with real patients being transported and undergoing AGPs.
As mentioned above, testing was not possible in the helicopter or jet with HVAC cabin air modulation due to high levels of environmental particles with the aircraft running. It is believed this creates a "worst-case scenario" in that no cabin air is exchanged, however, it is difficult to say with certainty how HVAC would have impacted results.

There were no reported or observed safety events during the use of the AerosolVE helmet. Although the study originally intended to deliver CPAP pressure at $10 \mathrm{~cm}$ of water and HFNC flow at $60 \mathrm{~L} /$ minute, due to participant discomfort, these were reduced to $5 \mathrm{~cm}$ of water and $30 \mathrm{~L} /$ minute, respectively.

\section{Conclusion}

Given the risk posed to prehospital medical providers by communicable respiratory diseases, development of novel devices to improve safety for these caretakers while still enabling use of respiratory therapies is of paramount importance. This holds true not only in the setting of a pandemic, but also during traditional "respiratory virus season" when a healthy workforce is critical to the function of the prehospital system. The AerosolVE Helmet demonstrated efficacy in creating a negative pressure environment around simulated patients and provided significant filtration of simulated respiratory droplets, thus making the confined space of various EMS transport vehicle types potentially safer for EMS personnel.
References

1. Pasnick S, Carlos WG, Dela Cruz CS, Gross JE, Garrison G, Jamil S. SARS-CoV-2 transmission and the risk of aerosol-generating procedures. Am J Respir Crit Care Med. 2020;202(4):13-14.

2. Cournoyer A, Grand'Maison S, Lonergan AM, et al. Oxygen therapy and risk of infection for health care workers caring for patients with viral severe acute respiratory infection: a systematic review and meta-analysis. Ann Emerg Med. 2021;77(1):19-31.

3. Prezant DJ, Lancet EA, Zeig-Owens R, et al. System impacts of the COVID-19 pandemic on New York City's emergency medical services. J Am Coll Emerg Physicians Open. 2020;1(6):1205-1213.

4. Brown A, Schwarcz L, Counts CR, et al. Risk for acquiring COVID-19 illness among Emergency Medical Service personnel exposed to aerosol-generating procedures. Emerg Infect Dis. 2021;27(9).

5. Murphy DL, Barnard LM, Drucker CJ, et al. Occupational exposures and programmatic response to COVID-19 pandemic: an emergency medical services experience. Emerg Med J. 2020;37(11):707-713.

6. Shukla V, Lau CSM, Towns M, et al. COVID-19 exposure among first responders in Arizona. J Occup Environ Med. 2020;62(12):981-985.

7. Heinzerling A, Stuckey MJ, Scheuer T, et al. Transmission of COVID-19 to health care personnel during exposures to a hospitalized patient - Solano County, California, February 2020. MMWR Morb Mortal Wkly Rep. 2020;69:472-476.
8. Centers for Disease Control and Prevention. Guidelines for environmental infection control in health-care facilities: recommendations of CDC and the Healthcare Infection Control Practices Advisory Committee (HICPAC). MMWR. 2003;52(No. RR-10):1-48.

9. Haas NL, Bassin BS, Kota S, et al. Rapid development of a novel portable negative pressure device. Int J Tuberc Lung Dis. 2020;24(7):737-739.

10. Bassin BS, Haas NL, Puls HA, Kota S, Kota SS, Ward KR. Rapid development of a portable negative pressure procedural tent. Int J Tuberc Lung Dis. 2020;24(7): 740-743.

11. Rajajee V, Williamson CA. Use of a novel negative-pressure tent during bedside tracheostomy in COVID-19 patients. Neurocrit Care. 2020;33(2):597-603.

12. Haas NL, Bassin BS, A Puls H, Ward KR. Use of a negative pressure procedural tent in the emergency department during the COVID-19 pandemic. Am J Emerg Med. 2021;48:354-356.

13. Bar-On YM, Flamholz A, Phillips R, Milo R. SARS-CoV-2 (COVID-19) by the numbers. Elife. 2020;9:e57309.

14. Correll D. This is the latest Air Force solution to transport COVID-19 patients aboard military cargo aircraft. 2020 May. www.airforcetimes.com/news/ coronavirus/2020/05/07/this-is-the-latest-air-force-solution-to-transport-covid19-patients-aboard-military-cargo-aircraft. Accessed August 2021. 